\title{
A Narrative Methodology to Recognize Iris Patterns By Extracting Features Using Gabor Filters and Wavelets
}

\author{
Shristi Jha ${ }^{1, \mathrm{a}}$, Sindhu B.M. ${ }^{1, \mathrm{~b}}$ and S.Venkatesan ${ }^{2, \mathrm{c}}$ \\ ${ }^{1}$ Pre-final Year, Department of Computer Science \& Engineering, Dayananda Sagar College of Engineering, Bangalore, India. \\ ${ }^{2}$ Professor, Department of Computer Science \& Engineering, Dayananda Sagar College of Engineering, Bangalore, India. \\ ashristij97@gmail.com, bsindhub1995@gmail.com, ${ }^{\mathrm{c}}$ selvamvenkatesan@gmail.com
}

\begin{abstract}
Iris pattern Recognition is an automated method of biometric identification that uses mathematical pattern-Recognition techniques on images of one or both of the irises of an individual's eyes, whose complex random patterns are unique, stable, and can be seen from some distance. Iris recognition uses video camera technology with subtle near infrared illumination to acquire images of the detail-rich, intricate structures of the iris which are visible externally. In this narrative research paper the input image is captured and the success of the iris recognition depends on the quality of the image so the captured image is subjected to the preliminary image preprocessing techniques like localization, segmentation, normalization and noise detection followed by texture and edge feature extraction by using Gabor filters and wavelets then the processed image is matched with templates stored in the database to detect the Iris Patterns.
\end{abstract}

Keywords: Gabor filters, Near Infrared, Gabor wavelet transform, Hough Transform, Iris patterns, Bit patterns, Hamming Distance

\section{Introduction}

Iris patterns are unique to every individual on earth" this concept was first proposed by Frank Burch in 1939. John Daugman working at university of Cambridge, England, developed and patented the first algorithms to perform iris recognition in 1991. These algorithms became widely licensed through a series of companies: IriScan (a start-up founded by Flom, Safir, and Daugman), Iridian, Sarnoff, Sensar, LG-Iris, Panasonic, Oki, BI2, IrisGuard, Unisys, Sagem, Enschede, Securimetrics and L-1, now owned by French company Morpho.Just as every human being has different fingerprints, so does the minute architecture of the iris exhibit variations in every subject examined. Its features represent a series of variable factors whose conceivable permutations and combinations are almost infinite. Iris recognition is an automated method of biometric identification that uses mathematical pattern-recognition techniques on video images of one or both of the irises of an individual's eyes, whose complex random patterns are unique, stable, and can be seen from some distance. Iris recognition uses video camera technology with subtle near infrared illumination to acquire images of the detail-rich, intricate structures of the iris which are visible externally. A key advantage of iris recognition,

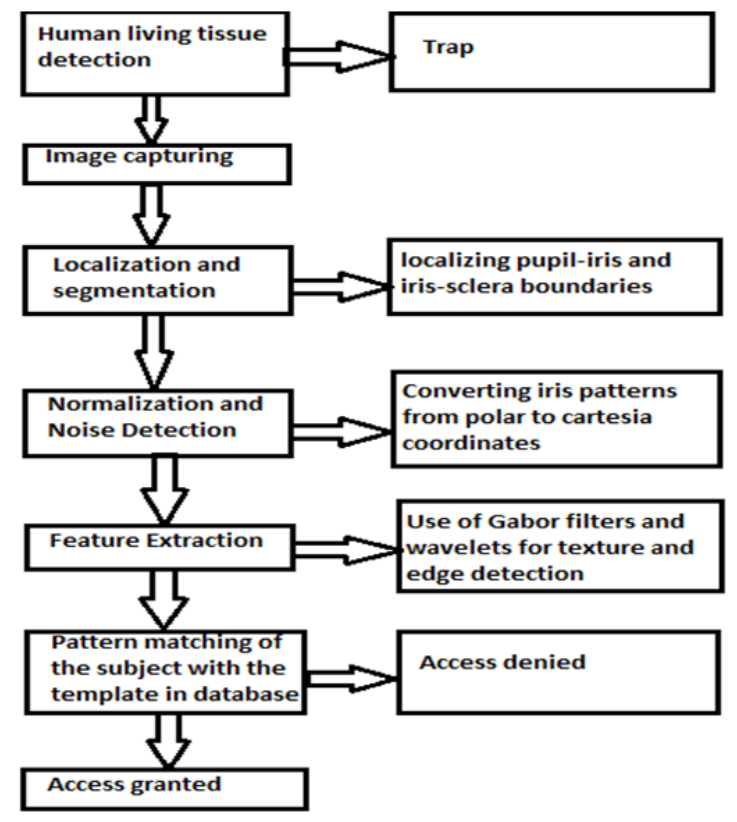

Figure 1. Work flow diagram

besides its speed of matching and its extreme resistance to false matches is the stability of the iris as an internal and protected, yet externally visible organ of the eye. The extraction of iris pattern has following steps: Image capturing, image segmentation, normalization, feature extraction and matching. Daugman [4] used multi scale quadrature wavelets to extract texture phase structure 
information of the iris to generate a 2048 bit iris code and compared the difference between iris representations by computing their hamming distance via the XOR operator.

Digital templates encoded from these patterns by mathematical and statistical algorithms allow the identification of an individual. Databases of enrolled templates are searched by matcher engines at speeds measured in the millions of templates per second per (single-core) CPU, and with remarkably low false match rates.

\section{Image Capturing}

Capturing an image of an iris is the initial stage of an iris based recognition system. Success rate of other recognition stages depends on quality of images taken. Images available in CASIA database do not have reflections in pupil and iris areas because NIR (700$900 \mathrm{~nm}$ ) is used for imaging. The majority of persons worldwide have "dark brown eyes", the dominant phenotype of the human population, revealing less visible texture in the Visible Wave length band but appearing richly structured, in the Near Infrared band(NIR). Using the NIR spectrum also enables the blocking of corneal specular reflections from a bright ambient environment, by allowing only those NIR wavelengths from the narrow-band illuminator back into the iris camera.

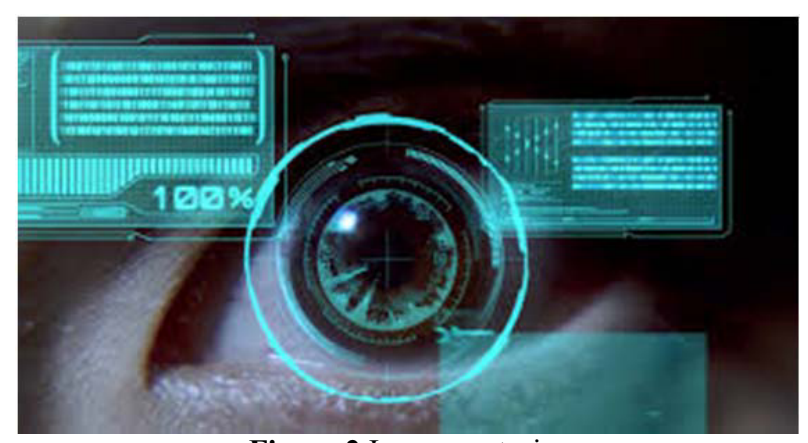

Figure 2.Image capturing

\section{Localization and Segmentation :}

Pupil is darkly pigmented compared to iris. Hence the use of canny edge Recognition results in determining points in iris pupil boundary. After determining the edge points by the use of circular Hogue transform the Centre and radius of iris circles are obtained. One of the important challenges in segmentation is detecting the boundary of iris and sclera. Illumination intensity distinction between iris and sclera is very low at the border; there are other edge points in the eye where illumination intensity is much more than that of the boundary of iris and sclera. As a result, edge Recognition algorithms which are able to detect outer iris edges identify those points as edge. Therefore, in order to detect iris outer boundary, these points have to be identified and eliminated. In this paper, available boundaries are initially enhanced and then extra edge points are identified and eliminated. At the end, through circular Hough transform, outer iris boundary is obtained. In order to enhance iris outer boundary edges, canny edge Recognition is performed on eye image in preprocessing stage.

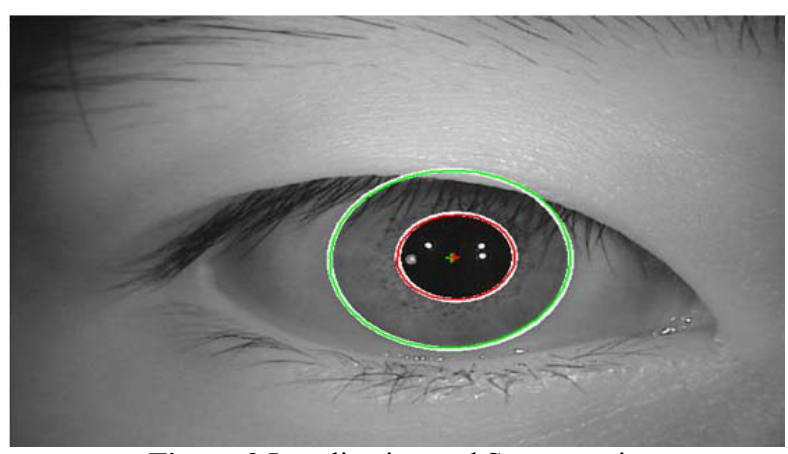

Figure 3.Localization and Segmentation

By performing such edge Recognition, a matrix is obtained with the same dimensions as of the image itself which its elements are high in areas where there is a definite boundary and the elements are low in areas where there is no perfectly definite boundary, such as iris outer boundary. Boundary between iris and eyelids can be differentiated as two lines. To localize them we use linear Hough transform[4]. Initially eyelid boundary should be detected by using canny edge Recognition. This method localizes eyelids with high precision by multiplying of 2.76 in matrix of pixel values of iris image and intensifying light in eye image, the edges are enhanced. The main disadvantage of this method is sclera boundary not being circular, is the result of angled imaging, and in these cases some information is lost.

\section{Normalization and Noise Detection}

Normalization is based on transforming iris areas from polar to Cartesian coordinates. This involves converting circular area into rectangle of equal area. Iris area is obtained as a strip with regard to iris boundary and pupillary center. 128 pupils centered circles are taken from iris pupil boundary and the pixels located on this circles are mapped into a rectangle. Pupil contracts or dilates with surrounding illumination level, so iris is not of the same size always. Hence choosing these 128 perfect circles normalize iris in terms of a rectangles of fixed sizes.

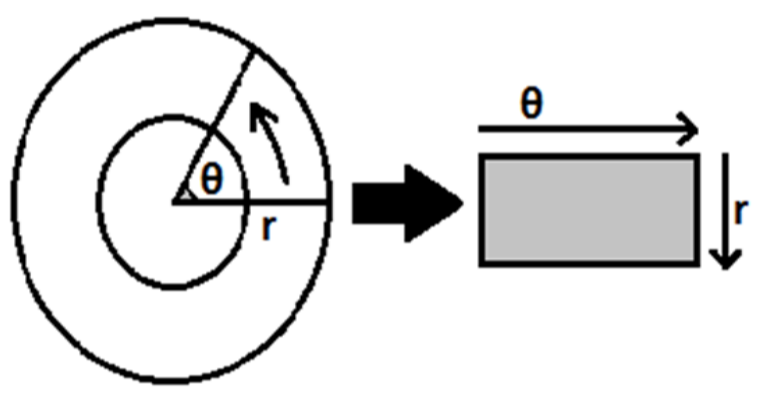

Figure 4.Normalization 
The set of pixels containing only the iris, normalized by a rubber-sheet model[3] to compensate for pupil dilation or constriction, is then analyzed to extract a bit pattern encoding the information needed to compare two iris images. An iris code is a 512 byte record. Any noises such as salt and pepper or cases where dark areas appear too dark are processed to get better image quality. Amplitude information is discarded so that the template is unaffected by light intensity.

Table 1: Accuracy rate of iris boundary localization for three databases [1]

\begin{tabular}{|l|l|l|l|}
\hline Database & $\begin{array}{l}\text { Accuracy } \\
\text { rate in } \\
\text { pupil } \\
\text { boundary } \\
\text { localizatio } \\
\mathrm{n}(\%)\end{array}$ & $\begin{array}{l}\text { Accuracy } \\
\text { rate in } \\
\text { sclera } \\
\text { boundary } \\
\text { localization } \\
(\%)\end{array}$ & $\begin{array}{l}\text { Accuracy } \\
\text { rate in } \\
\text { eyelids } \\
\text { boundary } \\
\text { localization } \\
(\%)\end{array}$ \\
\hline $\begin{array}{l}\text { CASIA Iris } \\
\text { Image } \\
\text { (ver. 1.0) }\end{array}$ & 98.13 & 99 & 98 \\
\hline $\begin{array}{l}\text { CASIA } \\
\text { Iris } \\
\text { Interval } \\
\text { (ver.3.0) }\end{array}$ & 99.73 & 99.19 & 98.63 \\
\hline
\end{tabular}

\section{Feature extraction:}

Gabor filters are used to extract features. In image processing, a Gabor filter, named after Dennis Gabor, is a linear filter used for edge Recognition [11]. Gabor filters can serve as excellent band-pass filters for onedimensional signals Frequency and orientation representations of Gabor filters are similar to those of the human visual system, and they have been found to be particularly appropriate for texture representation and discrimination. In the spatial domain, a 2D Gabor filter is a Gaussian kernel function modulated by a sinusoidal plane wave. Simple cells in the visual cortex of mammalian brains can be modeled by Gabor functions. Thus, image analysis with Gabor filters is thought to be similar to perception in the human visual system. Gabor wavelets are wavelets invented by Dennis Gabor using complex functions constructed to serve as a basis for Fourier transforms in information theory applications. Here is the formula of a complex Gabor function in space domain:

$$
g(x, y)=s(x, y) w_{r}(x, y)
$$

Where $\mathrm{s}(\mathrm{x}, \mathrm{y})$ is a complex sinusoid, known as the carrier, and $\mathrm{w} r(\mathrm{x}, \mathrm{y})$ is a 2-D Gaussian-shaped function, known as the envelope.

They are also closely related to Gabor filters; the important property of the wavelet is that it minimizes the product of its standard deviations in the time and frequency domain. Put another way, the uncertainty in information carried by this wavelet is minimized. Gabor filters are directly related to Gabor wavelets, since they can be designed for a number of dilations and rotations.
Usually, a filter bank consisting of Gabor filters with various scales and rotations is created. The filters are convolved with the signal, resulting in a so-called Gabor space. The Gabor space is very useful in image processing applications such as optical character recognition, iris recognition and recognition. The result of Gabor wavelet transform is a set of complex numbers that carry local amplitude and phase information about the iris pattern.

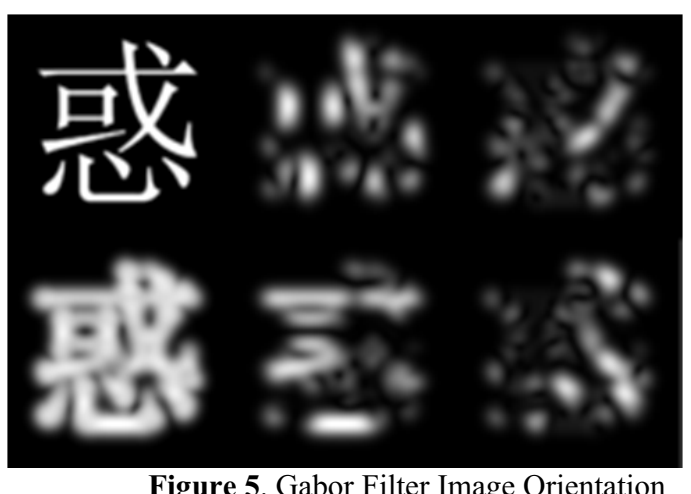

Figure 5 above shows the demonstration of Gabor filter: i.e, shown at 4 orientations at $0^{\circ}, 45^{\circ}, 90^{\circ}$ and 135 . The original image and superposition of all 4 images is shown on left.

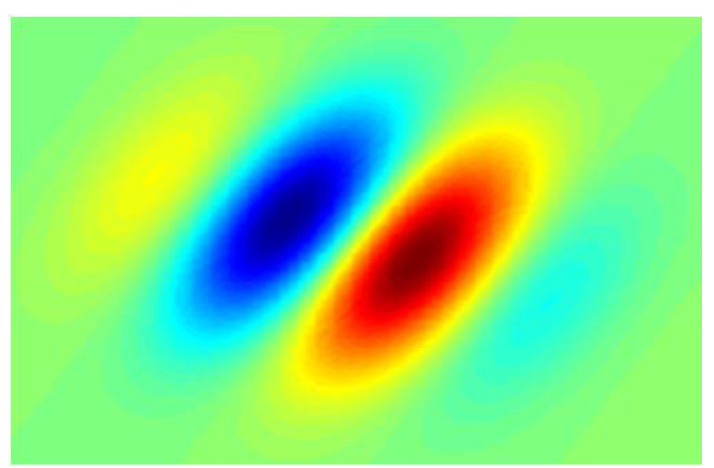

Figure 6. Image of Two dimensional Gabor filters

\section{Matching}

Most amplitude information is discarded, and the 2048 bits representing an iris pattern consist of phase information. Discarding the amplitude information ensures that the template remains largely unaffected by 


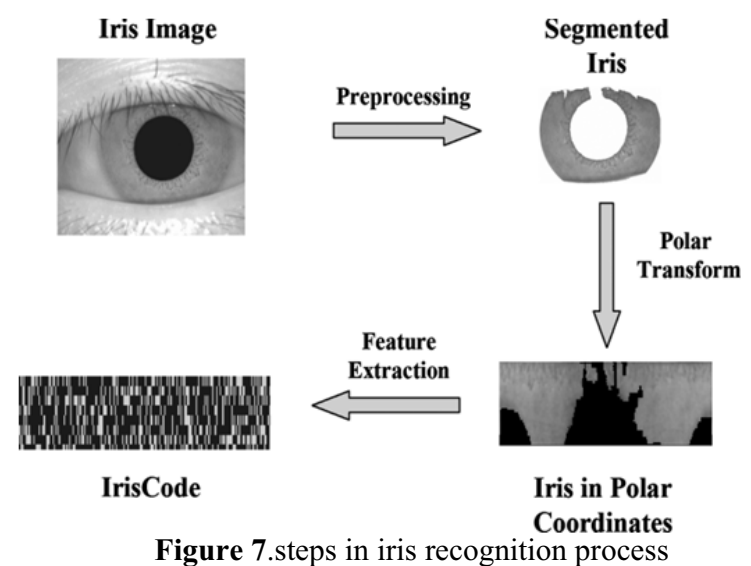

the changes in light illumination or camera gain and contributes to the long-term usability of the biometric template. For identification (one-to-many template matching) or verification (one-to-one template matching) a template created by imaging an iris is compared to stored template(s) in a database. If the Hamming distance is below the decision threshold, a positive identification has effectively been made (If a Hamming distance threshold of 0.26 is used, meaning that up to $26 \%$ of the bits in two Iris Codes are allowed to disagree due to imaging noise, reflections, etc., while still declaring them to be a match). Multi scale quadrature wavelets are used to extract texture, phase structure information of the iris to generate a 2048 bit iris code and compared with the template housed in database. The difference between iris representations is done by computing their hamming distance using the XOR operator. Table below gives truth table for the XOR. Considering the $\mathrm{n}^{\text {th }}$ bit of $\mathrm{r}$ and $\mathrm{s}$, we can know if they are similar or not:

Table 2: XOR truth table

\begin{tabular}{|ll|l|l|}
\hline $\mathrm{r}[\mathrm{n}] \mathrm{s}[\mathrm{n}]$ & $\begin{array}{l}\mathrm{d}(\mathrm{r}[\mathrm{n}], \mathrm{s}[\mathrm{n}])- \\
\text { distance between } \\
\text { digits }\end{array}$ & Similarity check \\
\hline 0 & 0 & 0 & similar \\
\hline 0 & 1 & 1 & dissimilar \\
\hline 1 & 0 & 1 & dissimilar \\
\hline 1 & 1 & 0 & similar \\
\hline
\end{tabular}

The distance is calculated as the number of bits of value 1 in the XOR result of the given two binary strings. This is the definition of the Hamming distance. For instance, if we consider two 8-bits vectors $\mathrm{R}$ and $\mathrm{S}$. The distance between them will be $\mathrm{dI}=\mathrm{I}(\mathrm{R} \oplus \mathrm{S})$ where $\oplus$ is the XOR operator and ' $\mathrm{I}$ ' is the function to calculate number of bits whose value is 1 in the binary XOR result. The mathematical algorithms allow the unique identification of a person by encoding digital templates from the iris patterns.

\section{Future Enhancement}

Iris scanners can be tricked using high resolution images of iris in place of a real eye. Iris scanning is susceptible to difference in light intensity and illumination. The remedy for this could be using spectral analysis instead of merely monochromatic cameras to distinguish iris tissue from other material; observing the characteristic natural movement of an eyeball testing for retinal retro reflection (red-eye effect) or for reflections from the eye's four optical surfaces (front and back of both cornea and lens) to verify their presence, position and shape. Some defense against the use of fake eyes and irises include changing lighting during the identification such that the pupillary reflex can be verified and the iris image be recorded at different pupil diameters.

A 2004 report from a German federal office stated that no live tissue Recognition technology was used for iris scanning, hence the use of sensors to detect live tissues or any of the above suggested methods could be used. The modern cataract surgery shows that it can change iris texture in such a way that iris pattern recognition is no longer feasible or the chance of falsely rejected cases is increased. Hence people with such surgeries must enroll a new request for iris scanning to government so that their database can be changed as early as possible. Consumption of alcohol may to lead to large dilation or contraction of the pupil, hence it would be advisable for the subject to restrain from alcohol at the time of verification.

The use of contact lenses is a major issue:

The verification rate with images from sensor and from cross-sensor is high and accurate in case of no contact lenses[10]. In case of soft lenses when it comes to sensor $47 \%$ of performance degradation is noted. In the case of matching images with no contact lens and images with textured contact lens, the verification rate is below $40 \%$ in all cases. The same situation holds for matching images with a soft lens against images with a textured lens. Hence it would be advisable to use a live tissue scanner to inform the subject to take the test without lenses.

Table 3: analyzing pattern recognition under lenses

\begin{tabular}{|l|l|l|}
\hline Lens type & Sensor & Cross-sensor \\
\hline No lens & Accurate results & Accurate results \\
\hline Soft lenses & Accurate results & $\begin{array}{l}47 \% \text { performance } \\
\text { degradation }\end{array}$ \\
\hline Textured lens & Not very & $\begin{array}{l}\text { Accuracy below } \\
\text { accurate }\end{array}$ \\
\hline
\end{tabular}

\section{Applications}

Some of these include:

Millions of persons around the world have been enrolled in iris recognition systems for convenience purposes such as passport-free automated border-crossings. Aadhaar ID program deployment began in 2011 in India, wherein the government is enrolling the iris patterns of all citizens for the Aadhaar scheme for entitlements distribution, run by the Unique Identification Authority of India (UIDAI).SRI International Sarnoff has been developing an "Iris on the Move" system for U.S. Government clients, capable of identifying 30 people per 
minute. Most recently, they have specialized in a product where drivers can be identified without needing to leave their vehicle. Since 2011, Google uses iris scanners to control access to their datacenters. Today, all of the UAE's land, air and sea ports of entry are equipped with iris recognition systems.

\section{Conclusion}

Iris Pattern Recognition has been done using the Gabor filters after the captured image is subjected to the image preprocessing techniques. After the result analysis it is found that through this novel methodology of extracting the features with respect to textures Recognition and discrimination with multiple orientation of the Gabor filter has given the best results in terms of Recognition rates of iris patterns. For identification of one to many template matching and for verification one to one template matching is preferred.

\section{References}

1. J.canny:a computational approach to edge Recognition IEEE transaction and pattern analysis and machine intelligence Vol 8(1986)[8] L. Flom and A. safir iris recognition system. U.S. patent no, 4641394 (1987)

2. R . P. Wildes, "Iris Recognition: An Emerging Biometric Technology", Proceeding of the IEEE, vol. 85, (1999), pp 1348-1363

3. J. Daugman, "How iris recognition works" ,IEEE Transactions on Circuits and Systems for Video Technology, vol. 14, pp. 21-30, (2002).

4. J. Daugman " How iris recognition works". Proceedings of 2002 International Conference on Image Processing, Vol. 1, (2002).

5. Chinese Academy of Sciences - Institute of Automation.Databas of 756 Greyscale Eye Images. http://www.sinobiometrics.com Version 1.0,(2003)

6. J. Daugman, "The importance of being random: statistical principles of iris recognition', Pattern Recognition 36 (2003) The Journal of the Pattern Recognition Society. Published by Elsevier Science Ltd.pp279-291

7. J .Huang ,H. Wechsler, visual rputines for eye loalisation using learning and evolution, IEEE Trrans .Evol.Comput.(4)(1)(2000)73-82

8. K. W. Bowyer, K. Hollingsworth and P. J. Flynn, "Image understanding for iris biometrics: A survey", Elsevier computer vision and Image Understanding, vol.110, (2008), pp. 281-307.

9. Z. Wei, X. Qiu, Z. Sun, and T. Tan, "Counterfeit iris Recognition based on texture analysis," in Proceedings of the 18th International Conference on Pattern Recognition, (2008). [10] Segmentation Techniques for Iris Recognition System, International Journal of Scientific \& Engineering Res V vol 2, Issue 4, April-2011, ISSN 2229-5518
IJSER C @ 2011 BY Surjeet Singh, Kulbir Singh.

10. Mahmoud Mahlouji and Ali Noruzi IJCSI International Journal of Computer Science Issues, Vol. 9, Issue 1, No 3, January[(2012)

11. PrateekVerma, MaheedharDubey, Praveen Verma, SomakBasu, International Journal of Emerging Technology and Advanced Engineering Website: www.ijetae.com (ISSN 2250-2459, Volume 2, Issue 6, June (2012)

12. SajidaKalsoom, Sheikh Ziauddin, COMSATS Institute of Information Technology Islamabad, Pakistan, The Fourth International Conferences on Pervasive Patterns and Applications, (2013).

13. DakshaYadav, Student Member, IEEE, NamanKohli, Student Member, IEEE, James S. Doyle, Jr., Student Member, IEEE, Richa Singh, Member, IEEE, MayankVatsa, Member, IEEE, Kevin W. Bowyer, Fellow, IEEE. SUBMITTED TO IEEE TRANSACTIONS ON IFS, OCTOBER (2013).

14. M. Vatsa, R. Singh and A. Noore, "Improving Iris recognition performance using segmentation, QualityEnhancement", Match Score Fusion, and Indexing, IEEE Transactions on Systems, Man, and Cybernetics B,Cybern., vol. 38, no. 4, pp 